\title{
$\begin{array}{ll}\text { Research Square } & \text { Preprints are preliminary reports that have not undergone peer review. } \\ \text { They should not be considered conclusive, used to inform clinical practice, }\end{array}$ or referenced by the media as validated information.
}

\section{The Network Structure of Resilience among Chinese Female Nursing Students}

\section{Yifei Wang}

Fourth Military Medical University

\section{Lin Wu}

Fourth Military Medical University

\section{Mei Wang}

Juxian Hospital of Traditional Chinese Medicine, Shandong Traditional Chinese Medicine University Cheng Sizhe

Fourth Military Medical University

\section{Zelong Yang}

Xi Jing Hospital, Fourth Military Medical University

\section{Lei Ren ( $\nabla$ rl_fmmu@163.com )}

Fourth Military Medical University

\section{Xufeng Liu}

Fourth Military Medical University

\section{Research Article}

Keywords: Resilience, Network analysis, Female, Nursing students

Posted Date: April 14th, 2021

DOl: https://doi.org/10.21203/rs.3.rs-402841/v1

License: (c) (i) This work is licensed under a Creative Commons Attribution 4.0 International License.

Read Full License 


\title{
The Network Structure of Resilience among Chinese Female Nursing Students
}

Yifei Wang ${ }^{1,} ;$ Lin $\mathrm{Wu}^{1,+}$; Mei Wang ${ }^{2}$; Cheng Sizhe ${ }^{1}$; Zelong Yang ${ }^{3}$; Lei Ren ${ }^{1, *}$; Xufeng Liu $^{1, *}$

${ }^{1}$ Department of Military Medical Psychology, Fourth Military Medical University,710032, Xi'an, China

${ }^{2}$ Department of infectious diseases, Juxian Hospital of Traditional Chinese Medicine, Shandong Traditional Chinese Medicine University, 276500, Rizhao, China

${ }^{3}$ Department of Hepatobiliary Surgery, Xi Jing Hospital, Fourth Military Medical University, 710032, Xi'an, China

*Correspondence: rl_fmmu@163.comlxf_fmmu@163.com

$\dagger$ Yifei Wang and Lin Wu contributed equally to this work

\begin{abstract}
Background: Many nursing students will suffer from serious psychological problem. However, the intervention research on the psychological resilience of nurses is still in the exploratory stage and the efficiency of existing intervention methods remains variable because of limited comprehension of this relevant psychological construct. Therefore this study investigates the network structure of the 10-item Connor-Davidson Resilience Scale (CD-RISC-10) in Chinese female nursing students to provide a novel understanding for resilience and targets for related interventions.
\end{abstract}


Methods: This study was an online cross-sectional study conducted at three medical universities from 21 August 2020 to 25 August 2020. This study adopted the CD-RISC-10, with a total of 776 participants joined in. Network analysis was conducted and the main focus is strength centrality and predictability.

Results: Three edges with strongest unregularized partial correlations existed in the final network, such as between item "Able to adapt to change" and item "Can deal with whatever comes". The items with strongest strength centrality are "Thinks of self as strong person" and "Can achieve goals despite obstacles".

Conclusions: The results may help us to gain a deeper understanding of resilience and provide educational orientation on how to make students more resilient, which may be benefit in challenge overcoming for nursing students.

Keywords: Resilience, Network analysis, Female, Nursing students 


\section{Background}

Resilience refers to the ability of an individual to maintain a good physical and mental state in a negative environment or power of recovery over difficult life experiences, and the ability to overcome problems experienced by individuals in poor environment and condition $[1,2,3]$. Resilience plays an important role in enabling nursing students to overcome a variety of challenges $[4,5,6]$. For instance, according to an Iranian qualitative study demonstrated that by increasing the levels of students' resilience, one can obtained a greater life satisfaction and get success more easily [7]. Previous studies have also shown that resilience may mitigate the adverse effects of stress and reduce the risk of depression in individuals with adverse childhood experiences $[8,9,10]$.

Nursing students are in a critical period of physical and mental development, many nursing students will suffer from endocrine disorders, emotional instability and other physical and mental symptoms faced with heavy academic burden, some even subject to depression, mania, and other serious psychological problem [11]. Moreover, in recent years, the conflicts between nurses and patients are increasingly intensified, and frequent violent incidents occur, which increases the fear of nursing students [12]. Of the estimated 43.5 million health care workers (HCWs) in the world, it is estimated that 20.7 million of those are nurses [13]. Initial data from a large sample $(n=1,257)$ in China suggest similar patterns, with half of the sample of front-line staff experiencing depression and anxiety during the COVID-19 Pandemic [14]. Therefore, much more attention should be paid to the enhancement of resilience in nursing education to cope with the mental health challenges faced by nurses in the future $[15,16]$. In recent years, there have been a few studies examining the use of interventions to improve psychological resilience of nurses or 
nursing students, such as mindfulness training for stress reduction training [17, 18], group positive psychological intervention method [19, 20], comprehensive intervention training [21]. However, the intervention research on the psychological resilience of nurses is still in the exploratory stage, no unified standard has been formed, the efficiency of different kinds of existing intervention methods remains variable because of limited comprehension of this relevant psychological construct and there is a great space for the development of the intervention research on the psychological resilience of nurses $[22,23]$.

Network approach is an important and innovative method which was put forward in the recent ten years. The method can also mathematically analyze and intuitively show the relationships between complex variables (usually regularized partial correlations) without relying on a priori assumption of causality between variables [24-25]. The existence of network approach provides another way to conceptualize mental constructs. According to the network approach, psychological constructs are considered as an interactive system, whose components interact with each other, and each component actively participates in the emergence of the psychological construct system, not just as a simple individual component [26]. At the same time, compared with the pure correlation method, the network analysis method is different in that it can be used to show the degree of centrality of the variable in the network. Central variables in psychological constructs can play a role in providing potential targets and targets for relevant interventions. In recent years, an increasing number of studies have been presented using network analysis methods to provide a network structure for related psychological constructs, such as well-being [26], self-worth [27], empathy [28], stigma [29], decision-making ability [30] and personality [31]. Specifically, a recent study also indicated that network model is a useful tool to explore resilience 
and identify targets for clinical interventions [32]. Therefore, it is reasonable to regard resilience as an interactive system based on this theory, which may provide a novel understanding for resilience and targets for related interventions.

In the present study, we adopted network analysis to investigate the network structure of 10-item Connor-Davidson Resilience Scale (CD-RISC-10) in Chinese female nursing students. We are particularly interested in how these items related to each other and the strength centrality of each item in order to provide a novel understanding for resilience in female nursing students and targets for related interventions.

\section{Methods}

\section{Settings and participants}

A cross-sectional study was conducted at three medical universities from From August 22nd to August $25^{\text {th }}, 2020$. The three colleges are Guangzhou University of Chinese medicine, Guangzhou Technical School, Rizhao Health School respectively. In this study, WeChat was used for the dissemination of our online survey, mainly drawing on the fact that WeChat is the most popular social media, with 1.15 billion active users in China [33]. A total of 798 Chinese nursing students from three medical universities participated in our study. All of these participants were undergraduate students majoring in nursing in the School of Nursing. In order to control the quality of the research and gender effect, twenty-two questionnaires were excluded due to their demographic information is incomplete or the respondents are males. At last, a total of 776 questionnaires were obtained.

\section{Survey instrument}


The Chinese version of the CD-RISC-10 employed in this study is a very common measurement and is mainly used for the investigation of psychological resilience. Besides, it has been confirmed to have good internal consistency (Cronbach's $\quad \alpha=0.862$ ) and excellent structure validity among Chinese nursing students, what' s more, the aggregated validity of this scale reached the statistical standard and could effectively express the validity of 25 items on the mental toughness scale, which could be applied in the nursing students [34]. Each item is rated on a 5-point scale from 0 to 4 (point referred to not true at all, rarely true, sometimes true, often true and true nearly all of the time, respectively). The sum of scores ranges from 0 to 40 , and the higher the total score, the better the level of person's resilience. The internal consistency of this scale in present study was excellent (Cronbach's $a=0.95)$.

\section{Ethical consideration}

The independent Ethics Committee of the First Affiliated Hospital of the Fourth Military Medical University approved the implementation of this study (number: KY20182047-F-1). The current study was an online survey through Wenjuanxing (www.wjx.cn) from 21 August 2020 to 25 August 2020. The first part of this online survey mainly included the informed consent. After reading the informed consent, participants can click "I agree" to complete the following survey if they want to further participate in this study. Next, they will complete the following items. Participants were also reminded that the survey was anonymous and personal information would not be disclosed, except for demographic data obtained in the first part.

\section{Data analysis}


Network analysis was used in this study. The networks were estimated via Gaussian graphical models (GGMs) [35]. GGMs are undirected networks in which the edges represent partial correlations between two nodes, after conditioning on all other nodes in the network. To account for the ordinal nature of the CD-RISC-10, the nonparametric Spearman rho correlations were used when estimating the network structure, as recommended by Epskamp and Fried [36]. Due to the low variables (10 variables) but high samples (776 individuals), we used unregularized model selection rather than regularization techniques commonly used in estimating GGMs [37]. The visualization of network was derived from the Fruchterman - Reingold algorithm, which locates nodes with stronger and more numerous connections near the center of the network and weakly associated nodes on the periphery [38]. In the visualized networks, blue edges represent positive correlations, and red edges represent negative correlations. Thicker edges mean stronger correlations between the nodes. The networks were constructed and visualized using the R-package qgraph [39].

Recent studies have shown that strength is the most reliable centrality index, and the centrality indices of betweenness and closeness seem especially unsuitable for assessing the importance of nodes in psychological networks [40-41]. Thus, we calculated strength of each node to assess and quantify the importance of each node by conducting the R-package qgraph [39]. Node strength is defined as the sum of the absolute value of the edge weights attached to a given node. Higher strength values indicate greater importance in the network. In addition, we computed the predictability of each node by using the R-package mgm [42]. Predictability is defined as the variance of a node which is explained by all its neighboring nodes, and this index could characterize the controllability of the network. 
We examined the robustness of network by using the R-package bootnet [35]. First, the accuracy of edge weights was evaluated by calculating $95 \%$ confidence intervals (CI) using a non-parametric bootstrap approach (2000 bootstrap samples). Second, the stability of node strengths was evaluated by computing correlation stability (CS) coefficient, using a case-dropping bootstrap approach. The value of CS-coefficient should not be below 0.25 and preferably should be above 0.5 [35]. Third, bootstrapped difference tests (2000 bootstrap samples and $a=0.05$ ) for edge weights and node strengths were performed to evaluate whether two edge weights or two node strengths differ significantly from one another.

\section{Results}

\section{Demographic characteristics}

There were in total 776 students participate in our study. The mean age of these patients was 18.87 \pm 0.95 years (mean $\pm \mathrm{SD}$, range 18-23 years). All of these participants were female, including 94 sole offspring, 682 non-sole offspring; 575 urban residents, 201 rural residents. The mean score of CD-RISC was $27.08 \pm 8.95$ (range $=0$-40). The descriptive statistics of each item of the 10 -item CD-RISC are shown in Table 1. In addition, the nonparametric Spearman rho correlation matrix of these items is shown in Table S1 (see Supplemental Materials).

Table 1 Descriptive statistics of each item of the CD-RISC-10

\begin{tabular}{lcccc}
\hline Item & $\mathrm{M}$ & $\mathrm{SD}$ & Stra & \\
\hline 1. Able to adapt to change (R1) & 2.72 & 1.04 & -0.74 & 0.57 \\
2. Can deal with whatever comes (R2) & 2.62 & 1.03 & 0.11 & 0.67
\end{tabular}




\begin{tabular}{|c|c|c|c|c|}
\hline 3. Tries to see humorous side of problems (R3) & 2.79 & 1.02 & 0.39 & 0.71 \\
\hline 4. Coping with stress can strengthen me (R4) & 2.88 & 1.05 & -0.76 & 0.65 \\
\hline 5. Tends to bounce back after illness or hardship (R5) & 2.52 & 1.31 & -2.12 & 0.38 \\
\hline 6. Can achieve goals despite obstacles (R6) & 2.74 & 1.04 & 1.20 & 0.75 \\
\hline 7. Can stay focused under pressure (R7) & 2.48 & 1.10 & 0.11 & 0.71 \\
\hline 8. Not easily discouraged by failure (R8) & 2.77 & 1.06 & 0.28 & 0.72 \\
\hline 9. Thinks of self as strong person (R9) & 2.80 & 1.06 & 1.25 & 0.78 \\
\hline 10. Can handle unpleasant feelings (R10) & 2.76 & 1.10 & 0.28 & 0.71 \\
\hline
\end{tabular}

Abbreviations: M, mean; SD, standard deviation; Str, Strength; Pred, predictability. a z-scores rather than raw centrality indices

\section{Network analysis}

The resilience network is shown in Fig.1a. There were several obvious characteristics in the network. First, $23(51 \%)$ edges are not zero among 45 possible edges and all these edges are positive. Second, three edges with strongest unregularized partial correlations existed between item R1 "Able to adapt to change" and item R2 "Can deal with whatever comes" (weight $=0.42$ ), between item R9 "Thinks of self as strong person" and item R10 "Can handle unpleasant feelings" (weight $=0.39$ ), between item R8 "Not easily discouraged by failure" and item R9 "Thinks of self as strong person" (weight $=0.35$ ). Third, the node predictability is shown as ring around node and ranges from 0.38 to 0.78 , with an average of 0.67 (see Table 1). Item R9 "Thinks of self as strong person" has the highest predictability: $78 \%$ of its variance can be explained by its neighboring nodes. Item R6 "Can achieve goals despite obstacles" has the second highest predictability: 75\% 
of its variance can be explained by its neighboring nodes. Item R5 "Tends to bounce back after illness or hardship" has the lowest predictability: $38 \%$ of its variance can be explained by its neighboring nodes.

The z-scored strength values for each node were calculated to assess their relative importance in the present network (see Table 1 and Fig. 1b). Two nodes with the highest strength are item R9 "Thinks of self as strong person" and item R6 "Can achieve goals despite obstacles", which indicates that these two nodes are the most associated nodes in the present network from a statistical point of view. The node with the lowest strength is item R5 "Tends to bounce back after illness or hardship", which indicates that this node is the least associated node in the present network from a statistical point of view.

\footnotetext{
$* * *$ Fig. $1 * * *$
}

Figure. 1. The network of Resilience.

Note: (a) Blue edge represented positive correlation, red edge represented negative correlation. The ring around nodes depicted its predictability. (b) Z-scored value of strength for each node. R1 $=$ Able to adapt to change; $R 2=$ Can deal with whatever comes; $R 3=$ Tries to see humorous side of problems; $R 4=$ Coping with stress can strengthen me; $R 5=$ Tends to bounce back after illness or hardship; $R 6=$ Can achieve goals despite obstacles; $R 7=$ Can stay focused under pressure; $R 8=$ Not easily discouraged by failure; $R 9=$ Thinks of self as strong person; $R 10=$ Can handle unpleasant feelings. 
Bootstrapped $95 \%$ confidence interval indicated that the estimation of edge weights was accurate (Figure S1 in the supplementary material). In addition, the CS-coefficients of node strengths was 0.75 , indicating that the estimation of node strengths is adequately stable (Figure S2 in the supplementary material). Bootstrapped difference test for edge weights showed that in the current network, a small to moderate proportion of the differences among edge weights were significant (Figure S3 in the supplementary material). Moreover, bootstrapped difference tests for node strengths showed that in the present network, a small to moderate proportion of the differences among node strengths were significant (Figures S4 in the supplementary material).

\section{Discussion}

In the present study, we adopted network analysis to investigate the network structure of 10-item Connor-Davidson Resilience Scale (10-item CD-RISC) in Chinese female nursing students. We are particularly interested in how these items related to each other and the strength centrality of each item in order to provide a novel understanding for resilience in female nursing students.

It is found that the three edges with the strongest connections are item R1 "Able to adapt to change" and item R2 "Can deal with whatever comes", between item R9 “Thinks of self as strong person" and item R10 "Can handle unpleasant feelings", and between item R8 "Not easily discouraged by failure" and item R9 "Thinks of self as strong person" respectively. The strong unregularized partial correlation between two nodes indicates that these two nodes have high co-occurrence. Item R1 "Able to adapt to change" indicates that a person is remarkably adaptable, No matter what the external environment looks like. Item R2 "Can deal with whatever comes" means that one can deal with a variety of different types of situations, no matter how varied or 
harsh the conditions are. From that point, we can see that item R1 "Able to adapt to change" and R2 "Can deal with whatever comes" have very similar expressions and may describe very similar concepts, so it is not surprising to find that they have a strong correlation. As for item R9 "Thinks of self as a strong person" and item R10 "Can handle unpleasant feelings", from a theoretical perspective, full affirming of oneself can become a wireless power to deal with various sources of stressful events and solve unpleasant things in work and daily life. When it comes to talking about the closely connection between item R8 "Not easily discouraged by failure" and item R9 "Thinks of self as a strong person", those who refuse to be defeated by failure easily can usually ignore setbacks and difficulties and can turn grief into strength, which encourages them to move forward and makes them more convinced that they can become a strong person. Besides, when person think they are strong enough, they will not easily allow themselves to be defeated by failure. It may be because confidence gives a person unlimited motivation and can make people more tenacious. The average predictability of the present network is $67 \%$, which suggested that the network consisted of items of CD-RISC-10 were more likely to be self-determined.

Node strength centrality may play an important role in finding items that activate or maintain psychological networks as well as providing potential targets for interventions. In other words, interventions aimed at item R9 "Thinks of self as strong person" and item R6 "Can achieve goals despite obstacles" may maximize the overall level of resilience in Chinese female nursing students. For a long time, nursing work has been considered as an emotionally demanding job, and nursing staff are often accompanied by anxiety ,burn-out and high levels of stress $[43,44]$. As for nursing students, they are in the crucial stage of learning. And they have not yet fully become a formal clinical workers, so they are somewhat weak in terms of professionalism and will usually show a 
lack of autonomy and the confidence, their knowledge reserves is corresponding weak. Besides, they would also be exposed to the challenges associated with medical environment, such as directly see patients experience pain and death [45]. Under the circumstance, resilience has been found to be correlated with the skills they need to cope with some kinds of difficulties and challenges. As mentioned earlier, the effectiveness of current interventions for resilience is limited, and the reason may be related to the lack of a comprehensive understanding of resilience. And as a result, it is difficult for us to find an valid target to adopt. On the basis of the above analysis, the network analysis conducted in this study shows and reveals the importance of these two nodes in psychological resilience. Therefore, we can surmise that the greatest benefits may be obtained through the intervention of item R9, "Think of yourself as strong", and item R6, "Achieve goals despite obstacles". It is thought that this may be an important complement to the limitations of the previous lack of a comprehensive and deep understanding of psychological resilience. Focusing on improving the personal qualities associated with self-confidence in R9 "Thinks of self as strong person" and R6 "Can achieve goals despite obstacles" in nursing education may enable them to better cope with various of challenges. For example, studies have shown that if teachers usually encourage nursing students to make them feel that they are good students and improve their self-confidence, the students would significantly improves their performance in nursing practice and work [46]. In addition, the predictability of item R9 "Thinks of self as strong person" is 0.78 , which suggests that this item is greatly influenced by its neighboring items in the network. This finding reminded that we could intervene on item R9 "Thinks of self as strong person" mainly by intervening on itself but also via its strong neighboring items (such as item R8 "Not easily discouraged by failure" and item R10 "Can handle unpleasant feelings") rather than via other 
related variables that are not included in the network. Meanwhile, the predictability of item R6 "Can achieve goals despite obstacles" is 0.75 , which suggests that we could intervene on item R6 "Thinks of self as strong person" mainly by intervening on itself but also via its strong neighboring items (such as item R7 "Can stay focused under pressure") rather than via other related variables that are not included in the network. In particular, it should be noted that predictability is the upper bound estimation.

There are several limitations in this study. First, since this study was a cross-sectional study, the causality between the variables could not be obtained through analysis. Second, the network in this study estimates between-subject effects on a group-level. Therefore, at the individual level, characteristics such as centrality and network structure can change dynamically. Third, in this network study, we only studied the variables contained in the scale we chose. As the results is strongly dependent on the variables, This study didn't include all of the most related aspects of psychological resilience.

\section{Conclusion}

In conclusion, this study is the first article investigating network structure of resilience in Chinese female nursing students. Results revealed three edges with the strongest connections and they are item R1 "Able to adapt to change" and item R2 "Can deal with whatever comes", item R9 "Thinks of self as strong person" and item R10 "Can handle unpleasant feelings", and item R8 "Not easily discouraged by failure" and item R9 "Thinks of self as strong person" respectively. Results also identify item R9 "Thinks of self as strong person" and item R6 "Can achieve goals despite obstacles" as potential targets for related interventions. The results may help us to gain a 
deeper understanding of resilience, guide the development of nursing students' resilience and provide educational orientation on how to make students more resilient, which may be benefit in challenge overcoming for nursing students. All the conclusion above needed further confirmatory studies to be validated.

\section{Ethics approval and consent to participate}

This study was conducted in accordance with the ethical standards put forth in the Declaration of Helsinki. Written informed consent was obtained from all individual participants included in the study. The study protocol was reviewed and approved by the Ethics Committee of Xijing Hospital, Fourth Military Medical University, Shanxi, China.

\section{Consent for publication}

Not applicable.

\section{Availability of data and materials}

The datasets used and/or analysed during the current study are available from the corresponding author on reasonable request.

\section{Competing interests}

The authors declare that the research was conducted in the absence of any commercial or financial relationships that could be viewed as a potential conflict of interest.

\section{Funding}


Not applicable.

\section{Authors' contributions}

YFW, LW and LR were responsible for the conception and study design. MW and ZLY performed the data collection. YFW, LW and SZC contributed to the analysis of the data. LR and XFL were involved in drafting the manuscript and revising it critically for important intellectual content. All authors have read and approved the final manuscript.

\section{Acknowledgments}

We would like to extend our sincere appreciation to the nursing students who participated in the study. We also thank all the administrative staff and teachers in the university who help us with the recruitment.

\section{Author information}

${ }^{1}$ Department of Military Medical Psychology, Fourth Military Medical University,710032, Xi'an, China, ${ }^{2}$ Department of infectious diseases, Juxian Hospital of Traditional Chinese Medicine, Shandong Traditional Chinese Medicine University, 276500, Rizhao, China, ${ }^{3}$ Department of Hepatobiliary Surgery, Xi Jing Hospital, Fourth Military Medical University, 710032, Xi'an, China

\section{References}


1. Bajaj, B., \& Pande, N. Mediating role of resilience in the impact of mindfulness on life satisfaction and affect as indices of subjective well-being. Personality and Individual Differences, 2016;93:63-67.

2. Deshields, T. L., Heiland, M. F., Kracen, A. C., \& Dua, P. Resilience in adults with cancer: development of a conceptual model. Psycho-oncology,2016;25(1):11-18. https://doi.org/10.1002/pon.3800

3. Öksüz, Y., \& Güven, E. The relationship between psychological resilience and procrastination levels of teacher candidates. Procedia-Social and Behavioral Sciences, 2014;116(1):3189-3193.

4. Sigalit, W., Sivia, B., \& Michal, I. Factors Associated With Nursing Students' Resilience: Communication Skills Course, Use of Social Media and Satisfaction With Clinical Placement. Journal of professional nursing : official journal of the American Association of Colleges of Nursing, 2017;33(2):153-161. https://doi.org/10.1016/j.profnurs.2016.08.006

5. Smith, G. D., \& Yang, F. Stress, resilience and psychological well-being in Chinese undergraduate nursing students. Nurse education today,2017;49:90-95. https://doi.org/10.1016/j.nedt.2016.10.004

6. Thomas, L. J., \& Revell, S. H. Resilience in nursing students: An integrative review. Nurse education today, 2016;36:457-462. https://doi.org/10.1016/j.nedt.2015.10.016

7. Abolghasemi, A. , \& Varaniyab, S. T. Resilience and perceived stress: predictors of life satisfaction in the students of success and failure. Procedia-Social and Behavioral Sciences, 2010;5(1):748-752. 
8. Sheerin, C. M., Lind, M. J., Brown, E. A., Gardner, C. O., Kendler, K. S., \& Amstadter, A. B. The impact of resilience and subsequent stressful life events on MDD and GAD. Depression and anxiety, 2018;35(2):140-147. https://doi.org/10.1002/da.22700

9. Poole, J. C., Dobson, K. S., \& Pusch, D. Childhood adversity and adult depression: The protective role of psychological resilience. Child abuse \& neglect,2017;64:89-100. https://doi.org/10.1016/j.chiabu.2016.12.012

10. Schulz, A., Becker, M., Van der Auwera, S., Barnow, S., Appel, K., Mahler, J., Schmidt, C. O., John, U., Freyberger, H. J., \& Grabe, H. J. The impact of childhood trauma on depression: does resilience matter? Population-based results from the Study of Health in Pomerania. Journal of psychosomatic research, 2014; 77(2): 97-103. https://doi.org/10.1016/j.jpsychores.2014.06.008

11. Guo, Y. F., Zhang, N., \& Zhang, J. P. The level and influencing factors of positive psychological qualities among nursing students. Chinese Journal of Nursing, 2013;48(01): 52-55.

12. Ye, Q. Q., Li, Y., Li, J. F., Zou, J. J., Ni, C. F., Wang, S. T., \& Zhang, S. S. Survey of influence of incident of violence between nurses and patients on nursing students in school. Chinese Nursing Research, 2016;30 (4A): 1224-1227.

13. World Health Organisation. (WHO, 2020)Health workforce nursing and midwifery. Available at: https://www.who.int/hrh/nursing_midwifery/en/. Accessed 23 April.

14. Albott, C. S., Wozniak, J. R., McGlinch, B. P., Wall, M. H., Gold, B. S., \& Vinogradov, S. Battle Buddies: Rapid Deployment of a Psychological Resilience Intervention for Health Care Workers During the COVID-19 Pandemic. Anesthesia and analgesia, 2020;131(1):43-54. https://doi.org/10.1213/ANE.0000000000004912 
15. McCann, C. M., Beddoe, E., McCormick, K., Huggard, P., Kedge, S., Adamson, C., \& Huggard, J. Resilience in the health professions: A review of recent literature. International Journal of Wellbeing, 2013; 3(1): 60-81.

16. Öz, F., Inci, F., \& Bahadir-Yilmaz, E. The death anxiety and resilience levels of nursing students and relevance between these levels. Yeni Symposium, 2012; 50 (4): 229-236.

17. Cheng, X. J., Qi, M. H., \& Lao, H. S. Effect of Mindfulness-base Stress Reduction on Negative State and Psychological Resilience of Women with Climacteric Syndrome. Medical Innovation in China, 2020;17(29):164-167. DOI:10.3969/j.issn.1674-4985.2020.29.041

18. Fortney, L., Luchterhand, C., Zakletskaia, L., Zgierska, A., \& Rakel, D. Abbreviated mindfulness intervention for job satisfaction, quality of life, and compassion in primary care clinicians: a pilot study. Annals of family medicine,2013;11(5):412-420. https://doi.org/10.1370/afm.1511

19. Xie, H. F., Ning, L., Xu, J., Wang, Y. M., Li, Y. M., \& Lu, J. The effects of group active psychological intervention on resilience of nursing students. Chinese Nursing Education, 2019;16(03):196-199. DOI:10.3761/j.issn.1672-9234.2019.03.007

20. Wu, M. The effects of Positive Psychology Group Intervention on Stress, Resilience and Subjective Well-being in Undergraduate Nursing Students. Bengbu Medical College,2019.

21. Mealer, M., Conrad, D., Evans, J., Jooste, K., Solyntjes, J., Rothbaum, B., \& Moss, M. (2014). Feasibility and acceptability of a resilience training program for intensive care unit nurses. American journal of critical care : an official publication. American Association of Critical-Care Nurses,2014; 23(6): e97-e105. https://doi.org/10.4037/ajcc2014747 
22. Xiao, S. P., W, G. X., \& Zhang, Y. F. Research progress on the intervention of mental toughness in nurses. Journal of Nursing(China), 2016;23(21):30-33.

23. Chmitorz, A., Kunzler, A., Helmreich, I., Tüscher, O., Kalisch, R., Kubiak, T., Wessa, M., \& Lieb, K. Intervention studies to foster resilience-A systematic review and proposal for a resilience framework in future intervention studies. Clinical psychology review,2018;59:78-100. https://doi.org/10.1016/j.cpr.2017.11.002

24. Borsboom, D., \& Cramer, A. O. Network analysis: an integrative approach to the structure of psychopathology. Annual review of clinical psychology,2013;9:91-121. https://doi.org/10.1146/annurev-clinpsy-050212-185608

25. Epskamp, S., \& Fried, E. I. A tutorial on regularized partial correlation networks. Psychological methods,2018;23(4):617-634. https://doi.org/10.1037/met0000167

26. Stochl, J., Soneson, E., Wagner, A. P., Khandaker, G. M., Goodyer, I., \& Jones, P. B. Identifying key targets for interventions to improve psychological wellbeing: replicable results from four UK cohorts. Psychological medicine,2019;49(14):2389-2396. https://doi.org/10.1017/S0033291718003288

27. Briganti, G., Fried, E. I., \& Linkowski, P. Network analysis of Contingencies of Self-Worth Scale in 680 university students. Psychiatry research,2019;272:252-257. https://doi.org/10.1016/j.psychres.2018.12.080

28. Briganti, G., Kempenaers, C., Braun, S., Fried, E. I., \& Linkowski, P. Network analysis of empathy items from the interpersonal reactivity index in 1973 young adults. Psychiatry research,2018;265:87-92. https://doi.org/10.1016/j.psychres.2018.03.082 
29. Wei, Z., Ren, L., Liu, C., Cao, M., Yang, Q., \& Deng, Y. The concept map of felt stigma in patient with epilepsy. Seizure,2020;80:138-142. https://doi.org/10.1016/j.seizure.2020.06.019

30. Peng, J., Ren, L., Yang, N., Zhao, L., Fang, P., \& Shao, Y. The Network Structure of Decision-Making Competence in Chinese Adults. Frontiers in psychology,2020;11:563023. https://doi.org/10.3389/fpsyg.2020.563023

31. Costantini, G., Richetin, J., Borsboom, D., Fried, E. I., Rhemtulla, M., \& Perugini, M. Development of indirect measures of conscientiousness: combining a facets approach and network analysis. European Journal of Personality, 2015;29(5), 548-567.

32. Briganti, G., \& Linkowski, P. Item and domain network structures of the Resilience Scale for Adults in 675 university students. Epidemiology and psychiatric sciences,2019;29,e33. https://doi.org/10.1017/S2045796019000222

33. Tencent. Tencent reported its fourth-quarter and full-year performance for 2019. 2020. Available at: https://cdc-tencent-com-1258344706.image.myqcloud.com/uploads/2020/03/18/19b5f2e87c7fc5a c6f173eccc723c040.pdf.

34. Ye, Z. J., Ruan, X. L., Zeng, Z., Xie, Q., Cheng, M. H., Peng, C. H., Lu, Y. M., \& Qiu, H. Z. Reliability and validity analysis of Chinese 10 -item resilience scale in nursing students. Journal of nursing, 2016;23(21):9-13.10.16460/j.issn1008-9969.2016.21.009

35. Epskamp, S., Waldorp, L. J., Mõttus, R., \& Borsboom, D. The Gaussian Graphical Model in Cross-Sectional and Time-Series Data. Multivariate behavioral research,2018;53(4):453-480. https://doi.org/10.1080/00273171.2018.1454823 
36. Epskamp, S., Borsboom, D., \& Fried, E. I. Estimating psychological networks and their accuracy: A tutorial paper. Behavior research methods,2018;50(1):195-212. https://doi.org/10.3758/s13428-017-0862-1

37. Williams, D. R., \& Rast, P. Back to the basics: Rethinking partial correlation network methodology. The British journal of mathematical and statistical psychology,2020;73(2):187-212. https://doi.org/10.1111/bmsp.12173

38. Fruchterman, T. M. J., \& Reingold, E. M. Graph drawing by force-directed placement. Software Pract. Exper,1991;21:1129-1164. https://doi.org/10.1002/spe.4380211102

39. Epskam, S., Cramer, A. O. J., Waldorp, L. J., Schmittmann, V. D., \& Borsboom, D. Qgraph: network visualizations of relationships in psychometric data. J Stat Softw,2012;48(4):1-18.

40. Epskamp, S., Rhemtulla, M., \& Borsboom, D. Generalized Network Psychometrics: Combining Network and Latent Variable Models. Psychometrika,2017;82(4):904-927. https://doi.org/10.1007/s11336-017-9557-x

41. Bringmann, L. F., Elmer, T., Epskamp, S., Krause, R. W., Schoch, D., Wichers, M., Wigman, J., \& Snippe, E. What do centrality measures measure in psychological networks? Journal of abnormal psychology,2019;128(8):892-903. https://doi.org/10.1037/abn0000446

42. Haslbeck, J., \& Fried, E. I. How predictable are symptoms in psychopathological networks? A reanalysis of 18 published datasets. Psychological medicine,2017;47(16):2767-2776. https://doi.org/10.1017/S0033291717001258

43. McGowan, J. E., \& Murray, K. (2016). Exploring resilience in nursing and midwifery students: a literature review. Journal of advanced nursing, 72(10), 2272-2283. https://doi.org/10.1111/jan.12960 
44. McVicar A. Workplace stress in nursing: a literature review. Journal of advanced nursing, 2003;44(6):633-642. https://doi.org/10.1046/j.0309-2402.2003.02853.x

45. Aradilla, H. A., Tomás, S. J., \& Gómez, B. J. Associations between emotional intelligence, depression and suicide risk in nursing students. Nurse education today,2014;34(4):520-525. https://doi.org/10.1016/j.nedt.2013.07.001

46. Chan, J. C., Sit, E. N., \& Lau, W. M. Conflict management styles, emotional intelligence and implicit theories of personality of nursing students: a cross-sectional study. Nurse education today, 2014;34(6):934-939. https://doi.org/10.1016/j.nedt.2013.10.012 

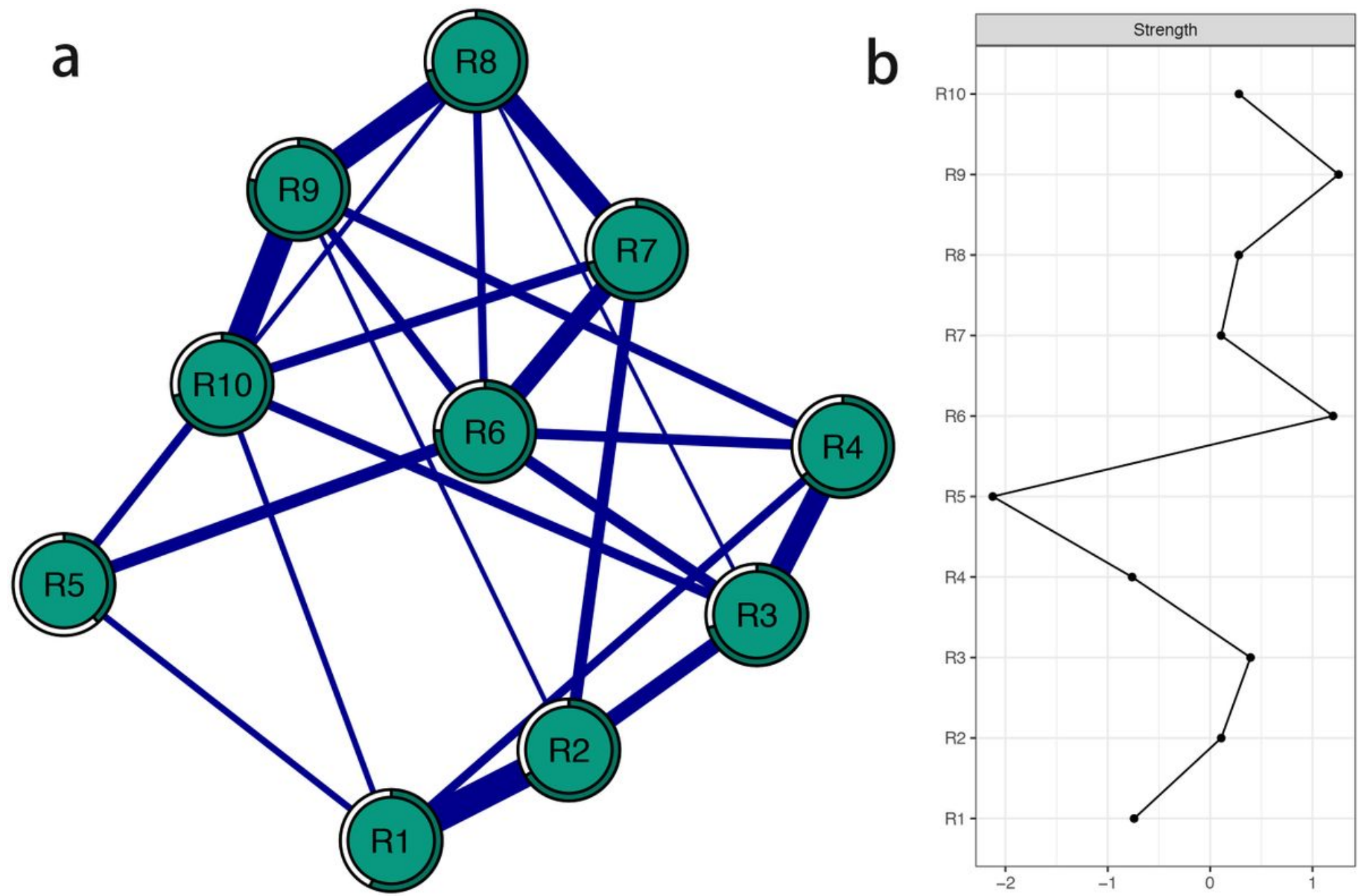

Figure 1

The network of Resilience. Note: (a) Blue edge represented positive correlation, red edge represented negative correlation. The ring around nodes depicted its predictability. (b) Z-scored value of strength for each node. $\mathrm{R} 1$ = Able to adapt to change; $\mathrm{R} 2$ = Can deal with whatever comes; $\mathrm{R} 3=$ Tries to see humorous side of problems; R4 = Coping with stress can strengthen me; R5 = Tends to bounce back after illness or hardship; R6 = Can achieve goals despite obstacles; R7 = Can stay focused under pressure; R8 = Not easily discouraged by failure; $\mathrm{R} 9=$ Thinks of self as strong person; R10 = Can handle unpleasant feelings.

\section{Supplementary Files}

This is a list of supplementary files associated with this preprint. Click to download.

- SupplementaryMaterials.docx 